\title{
Solving Major Problems Using Vector Affine Quantization
}

\author{
John R. Klauder \\ Department of Physics and Department of Mathematics, University of Florida, Gainesville, USA \\ Email: klauder@ufl.edu
}

How to cite this paper: Klauder, J.R. (2022) Solving Major Problems Using Vector Affine Quantization. Journal of High Energy Physics, Gravitation and Cosmology, 8, 178-183. https://doi.org/10.4236/jhepgc.2022.81013

Received: November 27, 2021

Accepted: January 7, 2022

Published: January 10, 2022

Copyright $\odot 2022$ by author(s) and Scientific Research Publishing Inc. This work is licensed under the Creative Commons Attribution International License (CC BY 4.0).

http://creativecommons.org/licenses/by/4.0/

\begin{abstract}
Affine quantization is a parallel procedure to canonical quantization, which is ideally suited to deal with special problems. Vector affine quantization introduces multiple degrees of freedom which find that working together creates novel tools suitable to eliminate typical difficulties encountered in more conventional approaches.
\end{abstract}

\section{Keywords}

Affine, Quantization, Vector Field, Models, Canonical Quantization

\section{An Introduction to Affine Quantization}

\subsection{Basics of Canonical and Affine Quantization}

The usual variables of classical physics are $p$ and $q$, and a common Hamiltonian is $H(p, q)=\frac{1}{2} p^{2}+V(q)$. If, for some reasons $q^{2}>0$, which is an example of our interest, then we prefer to use the dilation $d=p q$ and $q$. For them, the classical Hamiltonian becomes $H^{\prime}(d, q)=\frac{1}{2} d^{2} / q^{2}+V(q)$. However, canonical quantization (CQ) requires that $-\infty<p \& q<\infty$, and promotes its proper variables to operators, $p \rightarrow P\left(=P^{\dagger}\right)$ and $q \rightarrow Q\left(=Q^{\dagger}\right)$ with $[Q, P]=i \hbar \mathbb{1}$. Affine quantization $(\mathrm{AQ})$ chooses to promote proper variables to operators, $d=p q \rightarrow D=\left(P^{\dagger} Q+Q P\right) / 2\left(=D^{\dagger}\right), \quad q \rightarrow Q \neq 0$, and $[Q, D]=i \hbar Q$. The reason that $Q \neq 0$ is because if $Q=0$ then $D=0$ and $P$ cannot help. The fact that $Q \neq 0$ leads to either $Q>0, Q<0$, or simply both, $Q \neq 0$. Because $Q$ is "incomplete", the operator $P^{\dagger} \neq P$, and so $P^{\dagger}$ was used for $D$ so that $D^{\dagger}=D$. However, since $P^{\dagger} Q=P Q$, we find that $D=(P Q+Q P) / 2$ as well.

\subsection{An Example Where Affine Quantization Succeeds}

As an example of why affine variables are useful consider the well known har- 
monic operator Hamiltonian, $\mathcal{H}_{c q}=\left(P^{2}+Q^{2}\right) / 2=\left[-\hbar^{2}\left(\mathrm{~d}^{2} / \mathrm{d} x^{2}\right)+x^{2}\right] / 2$, which successfully uses CQ. However the half-harmonic oscillator, for which $Q>0$, requires $\mathrm{AQ}$. Since $P^{\dagger} \neq P$, the former Hamiltonian operator fails. Using $\mathrm{CQ}$, we are led to $\mathcal{H}_{0}=\left(P P^{\dagger}+Q^{2}\right) / 2$ AND $\mathcal{H}_{1}=\left(P^{\dagger} P+Q^{2}\right) / 2 .{ }^{1}$ These are TWO different, unequal, solutions, both of which came from the same classical Hamiltonian, $\left(p^{2}+q^{2}\right) / 2$. This is not acceptable quantization!

Instead, if the problem uses $\mathrm{AQ}$, and we insist that $q>0$, the classical Hamiltonian is $\left(p^{2}+q^{2}\right) / 2=\left(d^{2} / q^{2}+q^{2}\right) / 2$, and the new, and different, quantum Hamiltonian becomes

$$
\begin{aligned}
\mathcal{H}_{a q} & =\left(D Q^{-2} D+Q^{2}\right) / 2 \\
& =\left[-\hbar^{2}\left\{[x(\mathrm{~d} / \mathrm{d} x)+(\mathrm{d} / \mathrm{d} x) x] x^{-2}[x(\mathrm{~d} / \mathrm{d} x)+(\mathrm{d} / \mathrm{d} x) x]\right\} / 4+x^{2}\right] / 2 \\
& =\left[-\hbar^{2}\left(\mathrm{~d}^{2} / \mathrm{d} x^{2}\right)+(3 / 4) \hbar^{2} / x^{2}+x^{2}\right] / 2,
\end{aligned}
$$

which has the very same classical Hamiltonian [1], Section 1.5. While the full harmonic oscillator has equally spaced eigenvalues $\hbar[(0,1,2, \cdots)+1 / 2]$, the eigenvalues of the half-harmonic oscillator are $2 \hbar[(0,1,2, \cdots)+1]$, which shows a close physical connection between these two systems [2].

It should be clear that the mathematics of this example would permit us to not only solve the case for $x>0$, but also for $x<0$, and therefore, for this example, we could even display two different solutions at the same time!

This story is an example of how AQ has solved a problem that CQ cannot solve. The full harmonic oscillator is solved by CQ, and it fails using AQ. Evidently, each of these procedures can solve two different sets of problems.

Let us now introduce vector affine quantization (VAQ) as a lead-up to an illustration of a really difficult problem that VAQ can tackle.

\section{A First Look at Vector Affine Quantization}

Individual vectors have multiple similar elements such as $\boldsymbol{P}$ and $\boldsymbol{Q}$ for CQ systems. For AQ systems there is $D \equiv \frac{1}{2}\left(\boldsymbol{P}^{\dagger} \cdot \boldsymbol{Q}+\boldsymbol{Q} \cdot \boldsymbol{P}\right)\left(=D^{\dagger}\right)$ and $\boldsymbol{Q}\left(=\boldsymbol{Q}^{\dagger}\right) \neq 0$. For the same reason as before, $D=\frac{1}{2}(\boldsymbol{P} \cdot \boldsymbol{Q}+\boldsymbol{Q} \cdot \boldsymbol{P})$ is also correct. This particular set of basic operators is designed to force $Q \neq 0$, or stated otherwise as $\boldsymbol{Q}^{2}>0$. This requirement forces every component of $Q_{j}=0$, for $j=\{1,2,3, \cdots, N\}$ for a vector with $N$ components. But suppose we choose a different restriction such as $(\boldsymbol{Q} \cdot \boldsymbol{Q}-1) \neq 0$, which targets an $N$-dimensional sphere. This requires a different version of $D$. Since $D$ terms can be different, let us agree that a d-item pertains to a classical issue, and a D-item pertains to a quantum issue. So far we have only introduced D-items.

We now introduce the general scalar D-item to use that aspect along with ${ }^{1}$ Additional wrong candidate operators include $\left[\left(P^{\dagger}+P\right)^{2} / 4+Q^{2}\right] / 2,\left[\left(2 P^{\dagger}-P\right)\left(2 P-P^{\dagger}\right)+Q^{2}\right] / 2$, $\left[\left(P^{\dagger 4} / P^{2}+P^{4} / P^{\dagger 2}\right) / 2+Q^{2}\right] / 2$, etc., all having the same classical Hamiltonian! 
some $\mathcal{F}(\boldsymbol{Q}) \neq 0$, a scalar partner, such as $(\boldsymbol{Q} \cdot \boldsymbol{Q}-1)\left((\boldsymbol{Q} \cdot \boldsymbol{Q})^{2}-4\right)^{2} \neq 0$. Such examples need to be part of a $\mathrm{D}$-item leading to

$$
D=\frac{1}{2}\left[|\boldsymbol{P}|(\boldsymbol{Q} \cdot \boldsymbol{Q}-1)\left((\boldsymbol{Q} \cdot \boldsymbol{Q})^{2}-4\right)^{2}+\left((\boldsymbol{Q} \cdot \mathbf{Q})^{2}-4\right)^{2}(\boldsymbol{Q} \cdot \mathbf{Q}-1)|\boldsymbol{P}|\right],
$$

where $|\boldsymbol{P}|^{2}=\boldsymbol{P}^{\dagger} \cdot \boldsymbol{P}$. This example offers two spheres that have a common center with one sphere inside the other one. For this example, there are three distinct regions of interest: 1) outside of the largest sphere, 2) the region between the two spheres, and 3) inside the smallest sphere. As there were different options in Section 1.1, we can choose any one region, or any two regions, or all three regions to use as contributions to the quantization. If more than one region is accepted, continuity of coordinates between regions could be required.

We now turn to vector field theory models that can handle even bizarre vector affine quantization of such examples.

\section{Vector Affine Quantization of Complicated Field Theory Models}

An absurd vector model field theory, which is ripe for non-renormalizability using CQ, will be found formally soluble using VAQ. The purpose of studying this model is to show that if this "toy model" can be correctly formulated, then surely, less complex problems that are more physically relevant, can expect to have a proper formulation.

At first we introduce classical vector fields $\pi(x)$ and $\varphi(x)$ and propose an unusual classical Hamiltonian model. To make things more simple let us agree that $\pi(x)^{2} \equiv \pi(x) \cdot \pi(x)$ and $\varphi(x)^{2} \equiv \varphi(x) \cdot \varphi(x)$. Just to be crazy, let us choose $p$, the power of the interaction term as $10<p<\infty$, the number of spatial dimensions $s$ as $7<s<\infty$, and the coupling constant $g \geq 0$, all as part of the classical Hamiltonian,

$$
\begin{aligned}
H(\pi, \varphi)= & \int\left\{\frac{1}{2}\left[\pi(x)^{2}+\left(\nabla \varphi(x)^{2}\right)+m^{2} \varphi(x)^{2}\right]\right. \\
& \left.+g\left|\left(\varphi(x)^{2}-1\right)\left(\varphi(x)^{4}-16\right)\right|^{p}\right\} d^{s} x .
\end{aligned}
$$

While CQ could solve this problem if $g=0$, it could not solve it when $g>0$. Let us embark on our procedures using VAQ.

First we note an important feature about quantization of all such field theory problems that divergences do not arise because $\varphi(x)=\infty$. Instead, the field $\varphi(x)$ appears in some denominators that briefly vanish, and with large enough power, i.e., large $p$, integrations - think path-integration quantization-can yield divergencies. Our first job is to introduce a d-item, using $|\pi(x)|^{2}=\pi(x)^{2}$, and given by

$$
d(x)=|\pi(x)|\left(\varphi(x)^{2}-1\right)\left(\varphi(x)^{4}-16\right),
$$

which is a well-designed d-item. To protect $d(x)$ this process requires that both $\left(\varphi(x)^{2}-1\right) \neq 0$ and $\left(\varphi(x)^{4}-16\right) \neq 0$. These two equations define two spheres 
with the same center, and, in the present case, physics tells us to adopt all three sections of open regions.

Next, we modify the classical Hamiltonian to become

$$
\begin{aligned}
H^{\prime}(d, \varphi)= & \int\left\{\frac{1}{2} \llbracket d(x)^{2}\left[\left(\varphi(x)^{2}-1\right)\left(\varphi(x)^{4}-16\right)\right]^{-2}\right. \\
& \left.+\left(\nabla \varphi(x)^{2}\right)+m^{2} \varphi(x)^{2} \rrbracket+g\left|\left(\varphi(x)^{2}-1\right)\left(\varphi(x)^{4}-16\right)\right|^{p}\right\} d^{s} x,
\end{aligned}
$$

which now requires that the interaction term $\left|\left(\varphi(x)^{2}-1\right)\left(\varphi(x)^{4}-16\right)\right|^{p} \neq 0$. So far, this discussion has focused on the classical story. Now we turn to the quantum story.

The basic operators involved, again with $|\hat{\pi}(x)|^{2}=\hat{\pi}(x) \cdot \hat{\pi}(x)$, are

$$
\hat{D}(x)=\frac{1}{2}\left\{|\hat{\boldsymbol{\pi}}(x)|\left(\hat{\boldsymbol{\varphi}}(x)^{2}-1\right)\left(\hat{\boldsymbol{\varphi}}(x)^{4}-16\right)+\left(\hat{\boldsymbol{\varphi}}(x)^{4}-16\right)\left(\hat{\boldsymbol{\varphi}}(x)^{2}-1\right)|\hat{\boldsymbol{\pi}}(x)|\right\}
$$

and $\hat{\varphi}(x)$ has its several restrictions, namely $\hat{\varphi}(x)^{2} \neq 1$ and $\hat{\varphi}(x)^{2} \neq 4$. Note that $\hat{D}(x)$ can be zero thanks to $|\hat{\pi}(x)|=0$.

The quantum Hamiltonian is given by

$$
\begin{aligned}
\mathcal{H}^{\prime}(\hat{D}, \hat{\boldsymbol{\varphi}})= & \int\left\{\frac{1}{2} \llbracket \hat{D}(x)\left[\left(\hat{\boldsymbol{\varphi}}(x)^{2}-1\right)\left(\hat{\boldsymbol{\varphi}}(x)^{4}-16\right)\right]^{-2} \hat{D}(x)\right. \\
& +\left(\nabla \hat{\boldsymbol{\varphi}}(x)^{2}\right)+m^{2} \hat{\boldsymbol{\varphi}}(x)^{2} \rrbracket+g\left|\left(\hat{\boldsymbol{\varphi}}(x)^{2}-1\right)\left(\hat{\boldsymbol{\varphi}}(x)^{4}-16\right)\right|^{p} \mid \mathrm{d}^{s} x .
\end{aligned}
$$

First, it follows that

$$
0<\left\{\left|\left(\hat{\varphi}(x)^{2}-1\right)\right| \times\left|\left(\hat{\varphi}(x)^{4}-16\right)\right|\right\}^{-2}<\infty,
$$

and, for $1<p<\infty$, then it also follows that

$$
0<\left\{\left|\left(\hat{\varphi}(x)^{2}-1\right)\right| \times\left|\left(\hat{\varphi}(x)^{4}-16\right)\right|\right\}^{p}<\infty,
$$

a fact which guarantees that the quantization, although naturally quite complicated, would lead to a valid result, as it did for the half-harmonic oscillator.

\section{Why These Procedures Have Affine Roots}

Equation (4) can be reexamined as

$$
d(x)=|\pi(x)|\left(\varphi(x)^{2}-1\right)\left(\varphi(x)^{4}-16\right) \equiv|\pi(x)| w(x),
$$

in which $w(x)=0$ implies that $\left(\varphi(x)^{2}-1\right)\left(\varphi(x)^{4}-16\right)=0 .{ }^{2}$ The pair of classical variables, $d(x)=|\hat{\pi}(x)| w(x)$ and $w(x)$, for each $x$, are simply like $d=p q$ and $q$.

While classical variables for $\mathrm{CQ}$, namely $p \& q$, are promoted to quantum operators, it is required that they be Cartesian variables, specifically that $\mathrm{d} \sigma^{2}=A^{-1} \mathrm{~d} p^{2}+A \mathrm{~d} q^{2}$, in order to get physically correct operators [3]. On the ${ }^{2}$ It is somewhat extraordinary to imagine that removing a single point in one continuous function it automatically removes two whole spheres in another continuous function. 
other hand, to get physically correct quantum operators, the classical variables for $\mathrm{AQ}$, namely $d=p q$ and $q \neq 0$, are required to be from a constant negative curvature, such as $\mathrm{d} \sigma^{2}=B^{-1} q^{2} \mathrm{~d} p^{2}+B q^{-2} \mathrm{~d} q^{2}$, where the positive constant $B$ determines the magnitude of the curvature [1], Section 1.4. This relation requires that $q^{2}>0$, which then applies to either $q>0, q<0$, or both, $q \neq 0$.

For our principal topic, the classical variables, $d(x)=|\pi(x)| w(x)$ and $w(x) \neq 0$ must belong to constant negative curvatures to promote physically correct quantum operators, which arises from the metric

$$
\mathrm{d} \sigma^{2}=\int\left\{C(x)^{-1} w(x)^{2} \mathrm{~d}|\pi(x)|^{2}+C(x) w(x)^{-2} \mathrm{~d} w(x)^{2}\right\} \mathrm{d}^{s} x,
$$

in which $C(x)>0$ determines the magnitude of the constant negative curvatures for each $x$, and all of $w(x)>0$ and $w(x)<0$ are included in this specific analysis. The fact that our model has $(\nabla \hat{\varphi}(x))^{2}$ forces an effective continuity of $w(x)$ along spheres.

\section{Summary}

Canonical quantization tackles many field theory problems with a fixed procedure. On the other hand, this version of vector affine quantization allows for a carefully-designed operator retooling in order to tackle a large variety of Hamiltonians to ensure quantization succeeds as already was the case in properly quantizing the half-harmonic oscillator. Additional articles featuring affine quantization can be found in [4] [5] [6].

Having seen what procedures were used, the reader is welcome to carry out a VAQ of their own favorite, unsolved, quantum field theory problem.

\section{Conflicts of Interest}

The author declares no conflicts of interest regarding the publication of this paper.

\section{References}

[1] Klauder, J.R. (2020) Quantum Gravity Made Easy. Journal of High Energy Physics, Gravitation and Cosmology, 6, 90-102. https://doi.org/10.4236/jhepgc.2020.61009

[2] Gouba, L. (2021) Affine Quantization on the Half Line. Journal of High Energy Physics, Gravitation and Cosmology, 7, 352-365. https://doi.org/10.4236/jhepgc.2021.71019

[3] Dirac, P.A.M. (1985) The Principles of Quantum Mechanics. Clarendon Press, Oxford, 114.

[4] Klauder, J.R. (2020) The Benefits of Affine Quantization. Journal of High Energy Physics, Gravitation and Cosmology, 6, 175-185. https://doi.org/10.4236/jhepgc.2020.62014

[5] Klauder, J.R. (2020) Using Affine Quantization to Analyze Non-Renormalizable Scalar Fields and the Quantization of Einsteins Gravity. Journal of High Energy Physics, Gravitation and Cosmology, 6, 802-816. https://doi.org/10.4236/jhepgc.2020.64053

[6] Klauder, J.R. (2021) Using Coherent States to Make Physically Correct Classical-to- 
Quantum Procedures that Help Resolve Nonrenomalizable Fields Including Einstein's Gravity. Journal of High Energy Physics, Gravitation and Cosmology, 7, 10191026. https://doi.org/10.4236/jhepgc.2021.73060 